\title{
Starspots: signatures of stellar magnetic activity
}

\author{
Klaus G. Strassmeier \\ Astrophysikalisches Institut Potsdam, An der Sternwarte 16, D-14482 Potsdam, Germany \\ email: kstrassmeier@aip.de
}

\begin{abstract}
Starspots, just as Sunspots, are among the most obvious tracers and signatures of stellar surface magnetic activity. Emphasized already several decades ago as the origin for the rotationally modulated brightness of cool late-type stars, it is just now that we start to trace individual surface features in great enough detail to understand their magnetic behavior and interaction. Starspots also became the most important "noise" for detecting extra-solar planets and could possibly be decisive when it comes to detect another Earth. Since this is not a review, and because indirect imaging techniques are covered in other papers in this volume, I focus in this paper on some specific detections of starspots and introduce four new facilities particularly suited for starspot research in the near future.
\end{abstract}

Keywords. Magnetic fields - stars: spots - stars: magnetic fields - stars: rotation - methods: photometric - methods: spectroscopic - instrumentation: spectrographs - instrumentation: polarimeters

\section{Introduction}

Magnetic processes just like those seen in the space environment of the Earth and, of course, on the Sun have now slowly moved to center stage in other astrophysical areas. The application to other stars opened up a new field of research that became widely known as the solar-stellar connection. We emphasize that all previous attempts to disentangle the impact of magnetic fields onto stellar evolution had focused on higher-mass stars (see Maeder, this proceeding) or favored specific target classes in few subregions of the H-R diagram, e.g. Ap-stars like $\alpha^{2}$ CVn or 53 Cam, or T-Tauri stars (see Johns-Krull, this proceeding). Magnetic fields can play a crucial role in the accretion process as well as in the acceleration and collimation of jet-like flows in young stellar objects in general. Only just recently were, e.g., O-star magnetic-field measurements published (Wade et al. 2006, see also this proceeding). Finally, solar-type stars with convective envelopes and their rich but subtle variety of magnetic phenomena were added to the zoo just most recently (Catala et al. 2007, Donati et al. 2007, Petit, this proceeding).

Another area is the physics of accreting black holes, where magnetic activity is now believed to be responsible for most of the behavior of these objects, including their X-ray spectrum, their notoriously dramatic variability, and the powerful relativistic jets they produce. Another is the physics of the central engines of cosmical gamma-ray bursts, the most powerful explosions in the current universe. The main ingredient for explaining these events invokes powering and focusing of the bursts by magnetic fields. It is fair to say that virtually all the physics of magnetic fields exploited in these areas of astrophysics is based directly on our understanding of the Sun's magnetic field (see, e.g., Stenflo 2002, Solanki 2004, Rüdiger \& Hollerbach 2004, Thomas \& Weiss 2008). 

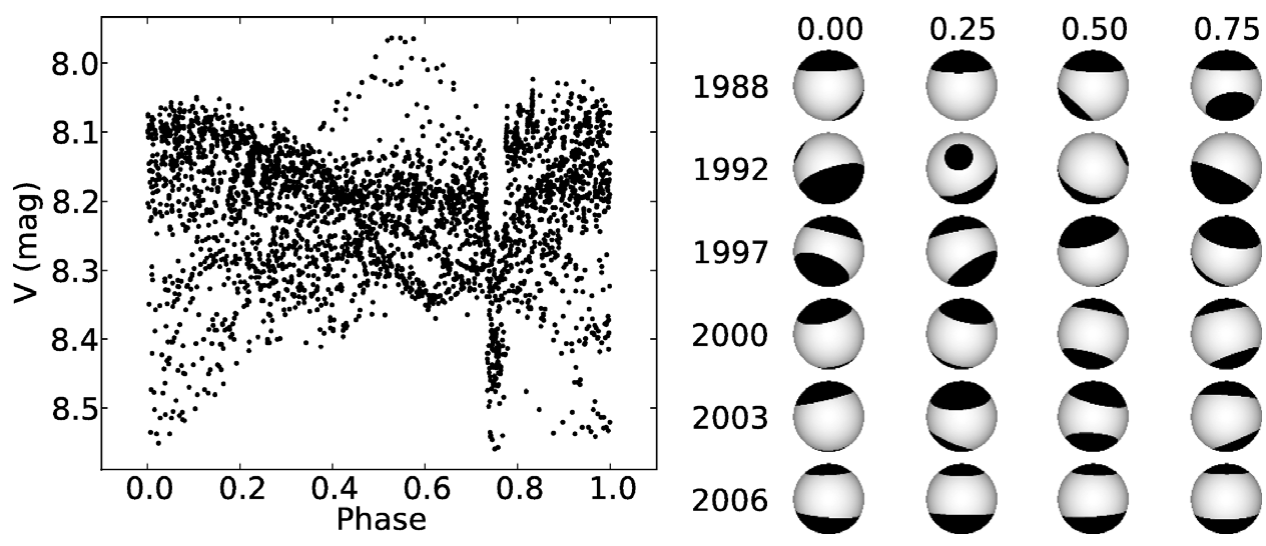

Figure 1. Almost all of the photometric variability of a magnetically active star is related to starspots. The light curve (left panel) of the spotted binary star HD 6286 shows 20 years of $V$-band data from the $0.75 \mathrm{~m}$ Vienna-T7 and the $0.40 \mathrm{~m}$ Tennessee-T3 Automatic Photoelectric Telescope, both located in southern Arizona. All data were phased with the rotation period of the primary component and demonstrate the continuous and systematic variability of the star as shown in the spot-model solutions to the right. Note that HD 6286 is an eclipsing binary with primary minimum at phase 0.75 .

\section{Starspot detections and some obvious limitations}

\subsection{Broad-band photometry}

Indirect detections and techniques, like the tracing and timing of sunspots moving across the solar disk due to rotation, led not only to the discovery of solar rotation but also to the discovery of the famous solar butterfly diagram. For stellar analogs, Vogt \& Penrod (1983) were the first to obtain an indirect image of a cool and heavily spotted star (V711 Tau = HR 1099) from data in late 1981 and discovered a prominent polar spot. Because the Sun does not show spots in excess of $\pm 40^{\circ}$ (but see Tsuneta 2008 for the recent discovery of high-latitude $\mathrm{kG}$ magnetic fields), this discovery spurred significant interest in these types of stars, and in starspots in particular. Today, dark photospheric spots and bright chromospheric plages are still the main tracers for detecting stellar rotation periods using photometry and, if long time series are available, also for detecting differential rotation and magnetic-cycle periods (Donahue et al. 1996, Rodonó et al. 2001, Strassmeier 2005).

Figure 1 is a recent data example and shows almost 20 consecutive years of automated $V$-band light curves of the spotted star HD 6286 (Strassmeier et al. 2008a). Plotting all data versus its 35-day rotation period is not a typical representation but is intended to emphasize the long-term variability due to changes of the star's spottedness. From this signal, systematic changes of the spot's surface positions, sizes, and possibly temperatures are derived and interpreted accordingly.

Figure 2 shows several simulated light curves and the stellar images obtained from them. Various spot configurations are adopted, as shown in the left columns, while the basic input parameters are kept fixed (see Savanov \& Strassmeier 2008 for details). Because photometric light curves represent only one-dimensional time series, opposite to spectral line profiles for Doppler imaging, the reconstructed stellar image contains only poor information in the direction of stellar latitude. Usually, the inversions easily resolve low-latitude details but practically fail to recover polar and high-latitude features. The code always tries to concentrate spots at the "sub-observer" latitude, where the photometric amplitudes become strongest. 

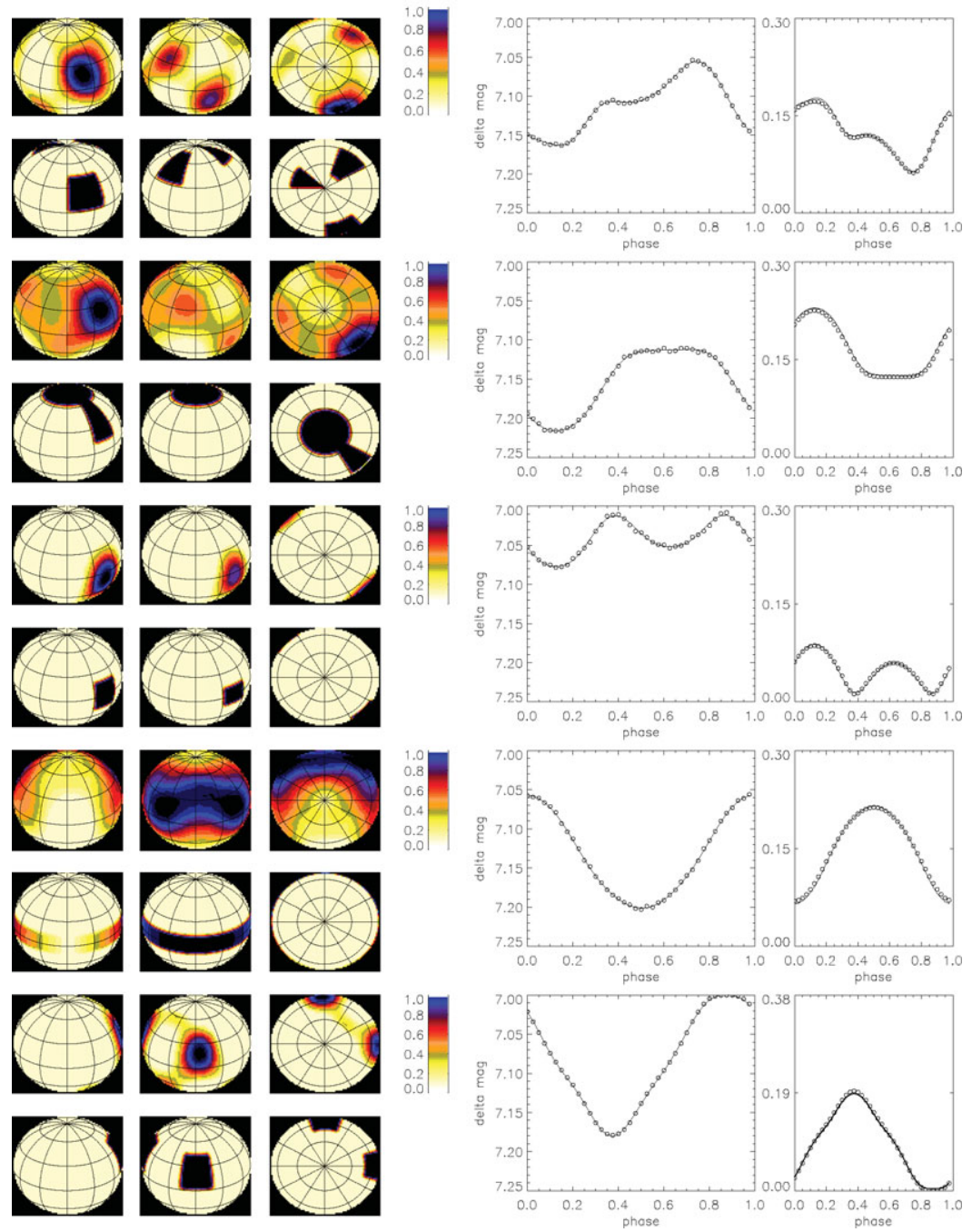

Figure 2. Light-curve inversions. The left columns show the image of the input test star and, above, its respective reconstruction. In the right columns are the simulated and reconstructed light curves and the change of the visible spottedness plotted versus rotational phase.

The tests in Fig. 2 illustrate another problem with the use of light curves. Two equal spots separated by $90^{\circ}$ in longitude produce a single symmetric light minimum, as would a single spot of slightly different shape. If such a light curve is used to search for "active longitudes" just by measuring the light-curve minimum, even the spot longitudes would be wrongly estimated while an inversion technique, as shown in Fig. 2, reproduces the correct longitudes.

\subsection{High-resolution spectroscopy and Stokes polarimetry}

A big deal was heard during this conference on spectropolarimetry. It appears clear now that full four-Stokes vector observations at high spectral resolution are the next future steps (replace high resolution with wavelength coverage for the class of degenerate stars)(see Carroll, this proceedings and Jordan, this proceedings). 
To reduce the complexity and the enormous computational requirements for even just Stokes-V Zeeman-Doppler imaging (ZDI) it became practice to use simplifying approximations in one part or the other during the course of the inversion. Whether these approximations are made in terms of a restrictive underlying stellar model (e.g. Donati et al. 1994), or applied to a simplified treatment of the polarized radiative transfer (e.g. Hussain 2000), or in terms of the all-present weak-field approximation, all these simplifications may severely limit the validity of the final result. The importance to accurately calculate and model the stellar spectra is of utmost importance for the interpretation of the observed polarized spectrum and the diagnostic of stellar magnetic fields (see Trujillo-Bueno, this proceedings).

The application of ZDI to molecular lines, in particular those with large Zeeman splittings in the near infrared, will finally give a first glimpse at the starspot magnetic field (see Berdyugina, this proceedings). Reconstructions from solely atomic lines are currently limited by the fact that the starspots themselves do almost not contribute to the overall ZDI signal. This leaves any inversion algorithm, with or without regularization, the choice of placing the magnetic field preferably at regions on the surface where there is also light, i.e. in bright features. As shown in the simulation by Carroll, this proceedings, one can bypass this to a certain extend by adding some artificial inversion weighting but, of course, one can not fool the data. However, exciting times are still ahead of us and I focus in this talk now on the new instrumental developments.

\section{New tools for starspot research}

In this section, I briefly introduce the projects relevant for starspot research that we are working on at AIP. This is biasing but, since this is not a review, it maybe useful for the reader. Starting with the already semi-operable STELLA observatory I will end with the, hopefully not so distant, European Extremely Large Telescope.

\subsection{STELLA}

STELLA consists of two robotic $1.2 \mathrm{~m}$ telescopes to simultaneously monitor stellar activity with a high resolution optical echelle spectrograph on one telescope, and a photometric imaging instrument on the other telescope (Weber et al. 2008). The STELLA observatory is located at the Observatorio del Teide on the Canary island of Tenerife. The STELLA Echelle spectrograph (SES) has been operated in robotic mode for two years now, and produced over 10,000 spectra of the entire optical range between 390 and $900 \mathrm{~nm}$ at a spectral resolution of 55,000 with a peak shutter-open time of 95\%. Although we do not use an iodine cell nor an actively stabilized chamber, its average radial velocity precision over the past two years was 60 to $150 \mathrm{~m} / \mathrm{s} \mathrm{rms}$, depending on target. The WideField STELLA Imaging Photometer (WIFSIP) is currently being tested and will enter operation in mid 2009.

\subsection{PEPSI for the $L B T$}

PEPSI is the fiber-feed high-resolution echelle spectrograph for the $11.8 \mathrm{~m}$ Large Binocular Telescope (LBT) in Arizona (Strassmeier et al. 2008c). It is designed to utilize the two $8.4 \mathrm{~m}$ apertures of the LBT in a unique spectropolarimetric mode. With two identical but independent polarimeters in each of the direct f/15 Gregorian foci, it will allow the simultaneous observation of circularly and linearly polarized light with high spectral and temporal resolution. Non-polarized "integral" light is fed to the spectrograph via two permanently mounted focal stations, thereby providing a standby spectrograph for quick-reaction science. Its thumbnail specifications are (see also Ilyin et al., this proceedings): 
- Fiber-fed optical integral-light spectroscopy in the wavelength range $380-1050 \mathrm{~nm}$ with spectral resolving powers, $R$, of 40,000 (2.2" aperture), 130,000 (1.5" aperture) and $310,000\left(0.75^{\prime \prime}\right.$ aperture). The $R=40,000$ mode with a sky aperture of $2.2^{\prime \prime}$ allows efficient spectroscopy even in bad seeing.

- Full wavelength coverage in three exposures. Pixel binning in dispersion direction can be used for $R=20,000$ work. $\mathrm{S} / \mathrm{N}$ of $10: 1$ of a $V=19 \mathrm{mag}$ star is reached at $R=130,000$ and seeing $0.7^{\prime \prime}$ in 1 hour integration time.

- Full four-Stokes polarimetry in the wavelength range 450-1050nm at a fixed spectral resolution of $\mathrm{R}=130,000$.

- Radial-velocity stability of order $\mathrm{m} / \mathrm{s}$ over an observing season in integral light.

- A polarimetric precision, $\delta P / P$ ( $P$ the degree of polarization), of $10^{-5}$ for targets brighter than $V=4 \mathrm{mag}$ in 1 hour integration time, of $10^{-3 \ldots 4}$ for targets brighter than $V=10$ th mag, $10^{-2}$ for $V<14 \mathrm{mag}$, and $10^{-1}$ for $V<18 \mathrm{mag}$.

- Fast reaction time for "Targets of Opportunity" due to the stand-by modus.

- The $R=310,000$ mode will be fed with Sunlight during daytime by a "Sun-as-a-star" telescope.

\subsection{GREGOR@night}

Gregor@night is the fiber-fed double echelle spectrograph (Strassmeier et al. 2007) for the night-time use of the new $1.5 \mathrm{~m}$ German solar telescope GREGOR. The telescope is foreseen to start routine day-time operation in 2010. The spectrograph's design specifications are driven by a science case based on the solar-stellar connection, in particular from the search and characterization of solar analogs but also ranging from the characterization of late-type stars with exoplanets to asteroseismology with high time resolution and cadence, and the long-term monitoring of stellar activity cycle analogs and related topics. The spectrographs are based on a white-pupil design with a $110 \mathrm{~mm}$ beam, two refractive cameras and collimators, both optimized for the wavelength ranges 360-490nm and 510-870nm for the two arms, respectively. The design achieves a two-pixel resolution of 100,000 for an entrance aperture of $3^{\prime \prime}$ at $20 \%$ total throughput. The instrument would be fully automated and no on-site night observer required.

\section{4. $I C E-T$}

ICE-T (the International Concordia Explorer Telescope) is a twin telescope for Dome C in Antarctica and consists of two $60 \mathrm{~cm} \mathrm{f} / 1.1$ ultra-wide-field Schmidt telescopes optimized for Sloan $g$ (402-552nm) and Sloan $i$ (691-818nm), respectively (Strassmeier et al. 2008b). The technical goal is to perform time-series photometry of a million stars between 9-18.5 mag. A photometric precision of up to 100 micro-mag for the brightest stars is envisioned. Such ultra-high precision for stars brighter than 10th mag is possible because the site has 3.6-times reduced scintillation which makes a $60 \mathrm{~cm}$ telescope in the Antarctic equivalent to a global network of $1.5 \mathrm{~m}$ telescopes at temperate sites. Nominal time resolution would be between 10 sec and 600 sec, depending upon the choice of co-adding and the on-site disk space. The two parallel-mounted $60 / 80 \mathrm{~cm}$ telescopes each feed a monolithic $10.3 \mathrm{k} \times 10.3 \mathrm{k}$ thinned, back-illuminated 9-micron CCD, operated in frame-transfer mode. Its total field of view (FOV) would be $12^{\circ}$ diameter, of which 65 square degrees would be seen by each $\operatorname{CCD}\left(8.1^{\circ} \times 8.1^{\circ}\right)$. The image scale is $2.75^{\prime \prime}$ per pixel with a nominal defocus to 6 pixels $\left(18^{\prime \prime}\right)$ for optimal point-spread-function sampling. Opposite to many other highly automated wide-field surveys, e.g. SDSS (Ivezić et al. 2004) or Pan-STARRS (Hodapp et al. 2004), ICE-T would operate in a truly robotic mode, i.e. with no human attendance at all. 


\subsection{SFPP: a Smart-Focal-Plane Polarimeter for the E-ELT}

We are currently conducting a design study of a spectropolarimetric light feed for the European Extremely Large Telescope (E-ELT) (Strassmeier \& Ilyin 2009). Such a polarimeter may feed an UV, an optical, and a near-IR spectrograph simultaneously, thus covering the 320-2500nm range. Our design concept is tentatively called SFPP, Smart Focal Plane Polarimeter. Its aim is to detect a differential polarimetric precision of $10^{-6}$ in selected spectral lines. The polarimeter itself shall be part of a smart focal plane for the symmetric beam of the telescope in its intermediate $\mathrm{f} / 4.15$ focus. Among the future scientific applications is a search for the linearly-polarized signal (or the lack thereof) from light reflected off a close-by planet in the combined Stokes spectra. We stress the importance of measuring stellar and planetary magnetic fields because these are prerequisites for planet habitability with life as we know it.

\section{Acknowledgements}

I would like to thank my colleagues Rainer Arlt, Thorsten Carroll and Ilya Ilyin for many discussions and Manfred Woche and Emil Popow for sharing their overall technical wisdom with me. I acknowledge grant STR645-1 from the Deutsche Forschungsgemeinschaft (DFG) and the support from the Bundeministerium für Bildung \& Forschung (BMBF) through the Verbundforschung grant 05AL2BA1/3 and 05A09IPA.

\section{References}

Catala, C., Donati, J.-F., Shkolnik, E., Bohlender, D., \& Alecian, E. 2007, MNRAS 374, L42

Donahue, R. A., Saar, S. H., \& Baliunas, S. L. 1996, ApJ 466, 384

Donati, J.-F., Achilleos, N., Matthews, J. M., \& Wesemael, F. 1994, A\&A 285, 285

Donati, J.-F., Moutou, C., Farés, R., et al. 2008, MNRAS 385, 1179

Hodapp, K. W., Kaiser, N., Aussel, H., et al. 2004, AN 325, 636

Hussain, G. A. J., Donati, J.-F., Collier Cameron, A., \& Barnes, J. R. 2000, MNRAS 318, 961 Ivezić, Z., Lupton, R. H., Schlegel, D., et al. 2004, AN 325, 583

Rodonó, M., Cutispoto, G., Lanza, A. F., \& Messina, S. 2001, AN 322, 333

Rüdiger, G. \& Hollerbach, R. 2004, The Magnetic Universe, Wiley-VCH, Berlin

Savanov, I. S. \& Strassmeier, K. G. 2008, AN 329, 364

Solanki, S. K. 2004, in G. Poletto \& S. T. Suess (eds.), The Sun and the Heliosphere as an Integrated System, A\&SS 317, p.373

Stenflo, J. O. 2002, in F. Moreno-Insertis, and F. Sánchez (eds.), Polarized radiation diagnostics of stellar magnetic fields, Astrophysical spectropolarimetry. XII Canary Islands Winter School, Cambridge University Press, p.55

Strassmeier, K. G. 2005, AN 326, 269

Strassmeier, K. G., Bartus, J., Fekel, F. C., \& Henry, G. W. 2008a, A\&\&A 485, 233

Strassmeier, K. G., Granzer, T., \& Denker, C., et al. 2008b, EAS Publications Series 33, 199

Strassmeier, K. G. \& Ilyin, I. V. 2009, in Moorwood, A. (ed.), Science with the VLT in the ELT era, Ap\&SS, Springer, p.255

Strassmeier, K. G., Woche, M., Granzer, T., Andersen, M. I., Schmidt, W., \& Koubsky, P. 2007, in Kneer, F., Puschmann, K. G., Wittmann, A. (eds.), Modern solar facilities - advanced solar science, Univ.-Verlag Göttingen, p.51

Strassmeier, K. G., Woche, M., \& Ilyin, I., et al. 2008c, SPIE 7014, 21

Thomas, J. H. \& Weiss, N. O. 2008, Sunspots and Starspots, Cambridge University Press

Tsuneta, S. 2008, in S. A. Matthews, J. M. Davis \& L. K. Harra (eds.), First Results From Hinode, ASP Conference Series, Vol. 397, p.19

Vogt, S. S. \& Penrod, G. D. 1983, PASP 95, 565

Wade, G. A., Fullerton, A. W., Donati, J.-F., Landstreet, J. D., Petit, P., \& Strasser, S. 2006, A\&A 451, 195

Weber, M., Granzer, T., Strassmeier, K. G., \& Woche, M. 2008, SPIE 7022 